\title{
Contrastive Studies of Pun in Figures of Speech
}

\author{
Qingchen Shao \\ Department of Foreign Language and \\ Art Design, \\ Cangzhou Vocational College of \\ Technology, \\ Cangzhou 061001, China \\ sqc0812@163.com
}

\author{
Zhenzhen Wang \\ College of Foreign Languages \\ Yanshan University \\ Qin Huangdao 066004, China \\ wangzhenzhencz@163.com
}

\author{
Zhijie Hao \\ Department of Information \\ Engineering, \\ Cangzhou Vocational College of \\ Technology \\ Cangzhou 061001, China \\ haozhijie@126.com
}

\begin{abstract}
Contrastive Studies of Pun in Figures of Speech. Pun as a figure of speech uses a word or phrase to get two meanings: literal meaning and connotation meaning so as to achieve humorous or sarcastic sense. There are many similarities between English and Chinese but in many ways they are different, while people always subconsciously consider them as equal and use them in any occasions. To solve these problems, this thesis solves them through the contrastive study of English and Chinese rhetorical figure-pun. By analyzing and comparing English and Chinese puns in some typical sentences and dialogues through semantic, pragmatic, cultural perspectives, partial tone, forms, meanings and so on. This thesis show that pun between English and Chinese exists lots of similarities and differences from different perspectives. Through all the endeavors done in this thesis, a better understanding of both languages and cultures, and smoother translation would be obtained. What's more, people can use puns in English and Chinese languages properly so as to eliminate unnecessary misunderstandings when communicating with others.
\end{abstract}

Keywords- pun contrastive study relevance theory cooperative principle differences and similarities

\section{INTRODUCTION}

All manuscripts must be in English. These guidelines include complete descriptions of the fonts, spacing, and related information for producing your proceedings manuscripts. Please follow them and if you have any questions, direct them to the production editor in charge of your proceedings at Conference Publishing Services (CPS): Phone +1 (714) 821-8380 or Fax +1 (714) 761-1784.

In the development of English language learning today, pun is defined as one of the most significant figures of speech, which is widely used in various literary works, for example the advertisements, poems, stories, novels and riddles, etc. And in both languages of English and Chinese, pun is always adopted because of its distinctive features in its nature and is always attempted to produce ambiguity as is planned so as to get the effect of expressing an implied meaning. The use of pun aims to target lots of effects: criticism, humor, or other kinds of the characters' thoughts and feelings, which can increase the charm of language and improve the beauty of the language so as to make readers feel more attracted. However, it is difficult to translate puns between English and Chinese, as the two distinctively different languages don't share much in common, especially in phonology and morphology which are the basic skills in the use of puns.

This paper attempts to solve the problem through the contrastive study of English and Chinese rhetorical figurepun, which is one of the basic methods for human beings to study and make understanding of things around us, and is also a way commonly adopted in linguistic studies. In 1994, Yang Zijian gave a definition for English-to-Chinese comparative linguistics at The National English-to-Chinese Comparative Research Assembly established in Changsha, and referred to it as a branch of linguistics, which has the property of both theoretical linguistics and applied linguistics. And its main task is to make a comparative study of synchronic and diachronic of English and Chinese languages, in order to make descriptions and expositions of differences and similarities between these two languages, and to apply these research achievements to theoretical language research and applied language research fields' . In 2000, Yang revised the last sentence of this definition as 'to apply these research achievements to language research and related research fields', which makes this definition more comprehensive, with the feature of multi-levels crossing several academic fields. Thus this paper expounds and draws principal distinctions and similarities of pun in English and Chinese, by using the contrastive study and analytical method.

\section{THEORETICAL FRAMEWORK}

The Collins Cobuild takes the use of pun as a tricky and wise strategy where the same pronunciation refers to two different meanings. For instance, if someone expresses 'The peasants are revolting', it constructs a pun because it contains two meanings: the peasants are doing things against authority or government, or that they are making others feel disgusted. The use of puns is adopted to give some kind of surprise and entertainment to others, or to express multiple meanings just with a single word or phrase. Looking at the same issue from different points of view, we may often come up with different categories of pun under discussion. But generally speaking, there are five categories of puns: 1) homophonic puns, 2) paronomasia, 3) grammatical puns, 4) sylleptic puns and 5) asterismus. Usually it has four major 
functions: 1) information conveying, 2) attention getting, 3) interest arising and 4) memory intensifying. In a word, the use of pun can lead to a humorous and clever effect with the contextual connection.

\section{A. Definitions of Puns in English and Chinese}

The study of rhetoric in the western world has been existed for long, ever since the ancient Greeks. And the study of rhetorical figures takes up significant place in the study of rhetoric, so it is of some contribution to research the rhetorical figures profoundly. It has a long history since rhetoric was researched. Aristotle and many scholars after him had brilliant contributions on rhetorical figures.

1)The Definition of Pun in English: The use of a word in such a way as to suggest two or more meaning or different associations, or the use of two or more words of the same or nearly the same sound with different meanings, so as to produce a humorous effect (Simpson and Weiner, 1989:1393)

2)The Definition of Pun in Chinese: The definition of pun in Chinese is:Pun in Chinese ---Shuang guan is one of rhetorical figures, using the relationship between words that may have the same sound to produce one sentence that involves in two things. In Chinese means pun 'two', or 'double', so in Chinese pun is related to 'two' kinds of sounds or meanings.

\section{B. Classifications of Pun in English}

Pun in English is usually classified into three categories-- paronomasia, antanaclasis and syllepsis Pun.

Paronomasia: Paronomasia is also called homophonic pun, which means the use of words with similar sound but different meanings. This kind of pun is often applied in literary works, humorous stories or advertisements to achieve the vivid, humorous or ironical effects. It usually can be divided into two categories:

Homophony refers to homophony of homomorphism which obtains various meanings.

E.g.1: He is not a grave man until he is a grave man.

In this sentence, the word 'grave' has been employed twice, with the same sound but totally different meanings. The first 'grave' used as an adjective, means 'serious'; while the second 'grave' used as a noun, means 'tomb'.

Approximant: It means the homophony of heteromorphy with different meanings.

E.g.2: On Sunday they pray for you and on Monday they prey on you.

'Pray' and 'prey' are pronounced the same sound here, but their meanings are entirely different. 'Pray' means 'speak (often silently) to God showing love, giving thanks, or asking for something'; 'prey' means 'steal from or plunder'. The sentence emphasizes on 'prey', which tries to make people against trusting those hypocritical prayers.

Antanaclasis: It refers to the use of a word twice or more, but with a different meaning for each time.

E.g.3: If we don't hang together, we'll hang separately.

The two 'hang' expresses totally different meanings. The first 'hang' means supporting one another; act in group. The second means 'to (cause to) die, especially in punishment, by putting with a rope around the neck'. 'We must be united' is indicated in this sentence.
Syllepsis Pun: It means this kind of pun adopting the use of a word, with two or more meanings.

E.g.4: He lost the game and his temper.

'Lose' is used once here, however, it has different meanings in 'lose the game' (fail to win, be defeated) and 'lose his temper' (become angry).

\section{C. Classifications of Pun in Chinese}

Pun in Chinese is usually classified into two categories--yu yi shuang guan (Semantic pun) and xie yin shuang guan (Homonymic pun).

Yu yi shuang guan(Semantic Pun)---a word could be used to express two meanings, and what have said and what want to say are very different.

E.g.5:She will see through the three Springs

And set no store

By the red of peach-blossom, the green of willows

Stamping out the fire of youthful splendor

To savor the limpid peace of a clear sky

A pun meaning of three springs is the three months of Spring and the three older China girls. In their names all the girls had the character of 'chun' or 'spring'.

Xie yin shuang guan(Homonymic Pun): It means words with the same or similar sound but quite different meanings.

E.g.6:Between the willows green the river flows along;

My gallant in a boat is heard to sing a song.

The west is veiled in rain, the east enjoys sunshine;

My gallant is as deep in love as day is fine.

In the Chinese poem '晴' and '情' have the same pronunciation 'qing', but it talks about the weather('qing' (晴) means 'clearing up') here. In fact it indicates the feeling and relationship between persons('qing' ( 情 ) means 'affection').

\section{Linguistic Motivation of Pun}

Relevance theory takes the place on the term of relevance together with the two principles of relevance: one is called the Cognitive Principle (that people would relate to the most relevant thought according to their cognition), and a Communicative Principle (that people would communicate in a way of maximum relevance).

1) Relevance Theory with Pun:

Relevance Theory takes a new view for puns. It makes it more important to understand the intention of one's utterance, and can make it more clear to separate between punning and ambiguity. There is asymmetry for communicative efficiency. "The fact that people and trees and elephants and cars all have trunks just proves that there are more things than there are words"(Redfern, 1982:7). Such asymmetry is necessary for efficiency in linguistic communication, yet it makes ambiguity more important - a word, phrase or sentence that has one or more meaning, could lead to misunderstandings or even fail the communication. Yet pun is often used on purpose in order to get the ambiguous nature and achieve certain effect in certain context. The punning word or phrase serves as a pivot to correlate two unrelated meanings into a unified entity and the role of receiver, according to Relevance Theory, is to come up with two different meanings in the cognitive environment of the speaker, and 
people who would recognize the true intention would discover the relevance between the two meanings in order to make a good communication. Thus it is significant to think beyond the words for what are implied in the sentences and make a clear understanding of the distinctive linguistic phenomenon.

E.g.7:

Zhou Fanyi: All right, you can go. The storm is coming around right now. (Murmuring and Pointing at the outside)

The most direct understanding of this sentence, which can be achieved by the receiver, is that the nature storm is approaching. But according to Relevance Theory, this kind of understanding rules out the possibility of the speaker's identification of the pun, because as the play develops, what Zhou really cares about is the intensifying contradiction. Through the optimal relevance, the receiver can figure out the underlying meaning of 'storm', and realize Zhou's real intention by extra cognitive efforts.

2) Cooperative Principles with Pun:

This principle is known as the COOPERATIVE PRINCIPLE or $\mathrm{CP}$ for short. To specify the $\mathrm{CP}$ further, Grice introduced four categories of maxims as follows:

1.QUANTITY

Make your contribution as informative as is required (for the current purposes of the exchange).

Do not make your contribution more informative than is required. true.

2. QUALITY :Try to make your contribution one that is

1. Do not say what you believe to be false. 2. Do not say that for which you lack adequate evidence.

3. RELATION: Be relevant.

4. MANNER: Be perspicuous.

1) Avoid obscurity of expression 2) Avoid ambiguity 3) Be brief (avoid prolixity) 4) Be orderly

The adoption of definitions such as 'principle' and 'maxim' does not refer that Communicative Principle and the rules are always obeyed in communication. Under certain context the rules on the other hand are purposefully disobeyed in order to get certain effect. It is to say that we usually and subconsciously know that we should obey the rules in communicating with others. We will utter information that are relevant, true, and informative enough, and also in clear and right manner. Also listeners would process what they heard in such a way. So when people speak in a way that violates the principle and maxims, it is always with an intention to around the listener's attention or interest.

In most cases, the rhetorical figure-pun usually violates Manner maxim in cooperative principle, because its obscurity and ambiguity of expression. It seems that the use of pun violates conversational maxims, but the inferring of its underlying meaning is subject to cooperative principle, and they can be known on the basis of some previous information. In his paper, Grice lists the necessary data like the followings:(1) the conventional meaning of the words used, together with the identity of any references that may be involved; (2) the CP and its maxims; (3) the context, linguistic or otherwise, of the utterance; (4) other items of background knowledge; and (5) the fact (or supposed fact) that all relevant items falling under the previous headings are available to both participants and both participants know or assume this to be the case(Hu Zhuanglin, 2006: 50)

Therefore, the speakers intend to violate conversational maxims in order to motivate the hearers to be concerned with the contextual side of meaning.

E.g.8: Benvolio: Here comes Romeo, here comes Romeo! Mercutio: Without his roe, like a dried herring. O flesh, flesh, how art thou fishified!

The word 'roe' has three senses: (a) the first syllable of Romeo's name; (b) deer (a pun on 'dear') and (c) reproductive organs of a male fish. Accordingly, Mercutio's words could be interpreted in the following three ways: (a) with 'Ro' reduced from his name, Romeo is left with nothing but 'me-O', the cry of a despairing lover; (b) The words could be understood as Mercutio teasing Romeo for his lovesickness - distracted without his 'dear' lover; (c) Mercutio develops the third sense of 'roe' and compares Romeo to be a dried herring with its reproductive organs removed.

\section{Similarities ANd Difference Of Pun In Both ENGLISH AND CHINESE}

In this chapter, I would like to explore the differences and similarities of pun in both English and Chinese languages. The emphasis should be put upon comparing the differences of pun in both English and Chinese from five perspectives. Since it is impossible to cover all the cultural motivations comprehensively, I will explore five typical aspects in detail.

First and foremost, I would like to sum up the similarities of pun in both English and Chinese as follows:

\section{A. Similarities of Pun in Both English and Chinese}

1) Similar Format and Rhetorical Function

Both English pun and Chinese pun are lexical rhetorical figures. From the definition we have talked about in the former chapter, some basic common features can be concluded:

- both puns in English and Chinese are a play on words, and their aim is to create a kind of amusing and humorous effect

- both puns in English and Chinese refer to the use of a word that has two or more meanings

- both puns in English and Chinese refer to the use of a word that has different associations

- both puns in English and Chinese refer to the use of two words that have the same sound while different meanings

- both puns in English and Chinese refer to the use of two words that have the similar sound while different meanings

2) Similar Formation Elements

Both puns in English and Chinese are based on polysemants and homonyms. The essential elements of creating puns are ambiguity and double contexts.

3) Similar Classification 
Such two categories as xie yin shuang guan and yu yi shuang guan in Chinese pun respectively correspond to those two branches--- paronomasia and antanaclasis in English pun. B. Differences of Pun in Both English and Chinese

For this part, I intend to make a comparatively detailed contrast of puns in both English and Chinese languages from the following different perspectives.

\section{1) Different Forms of Compositions}

Different Emphasis: From the definition and examples I have already provided above, we can find that the rhetorical figure---pun in Chinese language are broader and more exoteric. And xie yin shuang guan is used more frequently and flexibly, which has much relation to phonetic features of Chinese language. While the rhetorical figure---pun in English language focus on the language forms and use certain forms to express the rhetorical effect, the rhetorical figure---pun in Chinese stresses the language function to express the rhetorical effect. However, they have the same function to get the certain rhetoric effect.

Different Format: Pun in English is mainly made up of polysemy, while pun in Chinese is more dependent on context. In a certain context, a word or phrase that is relevant to two or more conceptions, ideas or feelings is often employed to make an expression more effective or humorous. This is achieved when there exists identity or similarity between certain words in such aspects as pronunciation, formation or meaning. This identity or similarity produces double meanings, which enables people to connect naturally the literal meaning with the implied meaning. Of these two meanings, the implied meaning is the principal part.

Antanaclasis makes use of the polysemy of certain words in certain context, and the original meanings of the words used to express the literal meaning will associate to the implied meaning.

E.g.9: We must all hang together, or we shall all hang separately.

In the sentence, the first 'hang' and the word 'together' give the meaning of being united as one and being concentrating (their) efforts on, while the second 'hang' means dance on a rope. The same word gives two totally different meanings in one simple sentence at the same time. It is hard to find Antanaclasis in pun in Chinese language taking repetition as its feature.

\section{2) Different Rhetorical Function}

I would like to illustrate this point of view from the following three perspectives:

First of all, the function of most puns in English is to produce humorous and amusing effect, but for pun in Chinese, only a small part has the effect of humor or amusement. Most puns in Chinese are used to motivate the hearer to explore the implied meaning of the speaker. Here I can give you more examples.

E.g.10: Customer: Waiter, will the pancakes be long?

Waiter: No, sir. Round.

Here in this case, the customer uses the word 'long' to mean whether he has to wait for a long time, but that is misunderstood by the waiter that the shape of the pancake is round not long, producing humorous effect.

\section{3) Different Psychological Motivation}

In traditional Chinese culture, collective interests take more priority to individual interests, so that the Chinese nation is more likely to conceal their true inner thoughts and feelings. They would rather express their feelings in a more roundabout and obscure way than in a direct way. However, in western culture, individuality is more important and paid much attention to. Therefore, puns in English are mainly used to achieve the concise and convictive way of expression, but in Chinese it focus more on ambiguity and implication of the original intention and motivates hearers to explore the sub audition, which means 'the words mean more than they say'.

E.g.11: English has always broken loose from the English, just as Latin broke loose from the Romans.

The first 'English' refers to the English language, while the second 'English' means the English people.

4) Different Cultural Background and Customs

Homophonic words in Chinese pun have much to do with their national customs and social prosperities, while Paronomasia in English pun lacks this kind of feature.

For instance, on Spring Festival, every household in China would like to put up the word 'fu' downwards to pray for good fortune, because the phrase 'fu dao', which means that good fortune is coming, has the same pronunciation with this phrase 'fu dao', whose meaning is that the word ' $f u$ ' is downwards. And this kind of xie yin shuang guan has been converted into national customs, and passed down from generations to generations. And this reflects that the Chinese nation has a stronger social mindset of tending to good luck and avoiding bad fortune.

What's more, it is impolite or even forbidden to send clocks, pears or umbrellas to friends or relatives as presents in many places around China, because the pronunciation of these words are almost the same or similar to those words representing unfortunate meanings. For example, the word 'clock' has the same pronunciation with the word 'death' (zhong) in Chinese language; 'pear' has the same pronunciation as 'being apart' (li); the word 'umbrella' has a similar pronunciation as 'separating' ( $\mathrm{san})$.

It is the cultural factors of different nations that cause some differences to puns in English and Chinese. There is one unique pun in Chinese called Chinese Allegories. This kind of pun in Chinese can represent the witness of Chinese language, wisdom of Chinese people and cultural mode of Han nation as much as possible. For example, Confucius removes ---full of books (lost); plug scallion into pig's nose--pretending to be elephant (disguising); the monk holding umbrella---not seeing hair and sky (lawlessness). Therefore it is understandable and reasonable for foreigners without Chinese cultural background not to appreciate this kind of pun.

\section{5) Other Cultural Differences}

Language is not only part of a culture but also the way to express a culture. Rhetorical figure reflects how the citizens live their life, construct a history, what they do with religions, and how are their values realized, how they think and express their politeness. Because of the cultural differences, the Chinese cannot quite understand English puns and 
receive the speaker's intention. And this also leads to the situation that English puns may mislead Chinese people.

Different History, Relative Stories and Characters: There exists a great difference in Chinese and Westerner's history. Confucianism takes control of Chinese thoughts, while Greece culture has great influence on westerners. For example, the advertisement of Radken hair salon is coming as follow:

Beauty is truth, truth beauty. - - Keats

Beauty made the bright world dims. - - Shelly

Lather. Rise. Repeat.—— Radken

If you know the English history, then you may as well know Keats and also the name of Shelly, because they are well-known British Romanticism poets. However, the lack of such knowledge may lead to misunderstanding of utterance and failure of communication. Thus the fact that we Chinese mostly don't understand other cultures will lead to failure of communication.

Different Religious Beliefs: Although China has many religions throughout the country, the most influential religions are Confucianism, Taoism and Buddhism. On the other hand, western countries are influenced by Greece mythology and Christianity. A lot of expressions from Christianity are walking into ads such as 'you don't have to be an angel to wear it.' Angel has been at the beginning served as the helper of God, but here it refers to kind and beautiful people.

Different Mode of Thinking and Social Values: In traditional Chinese culture, collective interests take more priority to individual interests, so that the Chinese nation is more likely to conceal its true inner thoughts and feelings. They would rather express their feelings in a more roundabout and obscure way than in a direct way. However, in western culture, individuality is more important and paid much attention to. In addition, the way of thinking for the Chinese is integrated and of curve type, while for westerners it is analyzed and of straight style. All these differences can have their representation and great influence on puns in both English and Chinese.

\section{CONCLUSION}

Rhetoric and rhetorical figures are essential in language, and it is hard to find passage without using rhetorical figures wherever in spoken language or in written from. It is said that rhetoric is the art of language considered as thought; grammar is the law of language considered as language. Grammar tells what is correct; rhetoric tells what is effusive and pleasing. Chinese and English belong to different language families, and there exist quite a lot differences. To achieve a smooth shift, the comparison between these languages is the first key step. Comparing the two languages from different layers, better understanding of both the language and the culture would be obtained, and a smoother and more suitable translation would be received. So through this thesis I would like to study the cultural differences from the perspective of figures of speech-pun by comparing puns in both Chinese and English languages. I believe it is helpful and useful for both the language speakers and the learners.

In conclusion, this thesis makes a contrastive study of puns in both English and Chinese language with the target to display their differences and similarities. By the exposition of definitions, categories and functions of puns in English and Chinese, and the analysis of linguistic accounts of this kind of rhetorical figure, the author intends to give the hope of enlightening and guiding English learners a bit in their language learning, translation studies, cross - cultural communication, etc.

To summarize, the study of rhetorical figure, by focus of the differences and similarities of puns between English and Chinese is not only interesting but also useful and of great significance. The promising area of study makes it clear that, though there are many boundaries to cross and barriers to overcome, achievements in this area would make it worth of all efforts to do study in the area of puns between English and Chinese language.

\section{REFERENCES}

[1] Bandar, Robert G. 1983. American English Rhetoric. New York: Holt, Rinehart and Winston.

[2] Brooks, Cleanth \& Robert Penn, Warren. 1979. Modern Rhetoric. New York: Harcourt Brace Jovanovich, Inc.

[3] Edward, P J, Corbett. 1990. Classical Rhetoric for the Modern Student, Camgridge: Camgridge University Press.

[4] J, Cuddon.1986. A Dictionary of Literature Terms, Oxford: Oxford University Press.

[5] Robert W., Daniel. 1967. A Contemporary Rhetoric, Boston: Little, Brown and Company.

[6] P. J., Edward \& Corbett, Robert J. Connors : Classical Rhetoric f or the Modern Student (Forth Edition), Oxford: Oxford University Press.

[7] Nida, Eugene. 1993. A. Language, Culture, and Translation. Shang Hai: Shang Hai Foreign Language Education Press.

[8] Richards, Jack C. \& Platt. John. 2000. Longman Dictionary of Language Teaching \& Applied Linguistics. Beijing: Foreign Language Teaching and Research Press.

[9] Richards, Ivor A. 1936. The Philosophy of Rhetoric. Oxford: Oxford University Press.

[10] Redfern, W. 1982. Puns: More Senses Than One. London: Penguin Books Ltd.

[11] Richards, Ivor. 1936. The Philosophy of Rhetoric. Oxford: Oxford University Press.

[12] Ruse, C. \& Hopton, M. 1992. The Cassell Dictionary of Literary and Language Terms. Beijing: Foreign Language Teaching and Research Press.

[13] Sherzer, J. 1978. Oh! That's a Pun and I didn’t know it. Semiotica. New Jersey: RPentice hall, INC.

[14] Simpsan, J. A. \& Weiner E S C. 1989. The Oxford English Dictionary. Oxford: Oxford University Press.

[15] Zeiger, J. 1978. An Encyclopedia of English. New York: Arco Publishing Company, Inc. 